Artículos

\title{
Validación y adaptación al español del cuestionario de relación médico-paciente en pacientes de diabetes mellitus tipo 2 en el norte de México (PDRQ-9)
}

\author{
Spanish Validation and adaptation of the Doctor-Patient Relationship Questionnaire (PDRQ-9) in
} type 2 diabetes mellitus patients in Northern Mexico

Karla Nathalia Fernández Castillo; Nadia Saraí Corral Frías; Edgar Rene Valenzuela Hernández

Universidad de Sonora

\section{Resumen}

La relación entre médico y paciente es esencial para la adherencia al tratamiento, sobre todo en padecimientos crónicos como lo es la diabetes mellitus tipo 2. El objetivo de la presente investigación fue validar el cuestionario Patient-Doctor Relationship Questionnaire (PDRQ-9) en una población mexicana, específicamente en el noroeste de México. El estudio fue transversal, donde participaron 307 pacientes con diabetes mellitus tipo 2 que acudían a un hospital público. EI PDRQ-9, así como un cuestionario de adherencia al tratamiento, fue respondido de manera individual a través de un dispositivo electrónico dentro de la sala de espera del centro de salud. Para investigar consistencia interna y validez, se realizaron análisis de fiabilidad y análisis factorial confirmatorio. Nuestros resultados demostraron que la escala tuvo una fiabilidad aceptable $(\alpha=0.95)$. El análisis factorial confirmatorio, usando el método de máxima verosimilitud, mostró que el modelo de un factor hipotetizado tuvo buen ajuste a los datos. Todos los ítems presentaron pesos de medida aceptables $(\lambda>0.5)$. La adaptación del PDRQ-9 en el noroeste de México reprodujo la estructura factorial encontrada en el estudio original y demostró una alta consistencia interna. Este estudio sugiere que el PDRQ-9 es un instrumento válido y confiable, útil para la medición de la relación médicopaciente en pacientes con diabetes mellitus tipo 2.

Palabras clave: relación médico-paciente, psicometría, validación, diabetes, adaptación

Karla Nathalia Fernández Castillo Universidad de Sonora

Nadia Saraí Corral Frías Universidad de Sonora

https://orcid.org/0000-0002-1934-0043

Edgar Rene Valenzuela Hernández Universidad de Sonora

Autor para correspondencia: nathaliafernandez93@gmail.com 


\begin{abstract}
The doctor-patient relationship is an essential predictor of treatment adherence, especially in those diagnosed with a chronic condition such as Type 2 Diabetes Mellitus. The aim of the present investigation was to validate the PatientDoctor Relationship Questionnaire (PDRQ-9) in a Mexican sample, specifically in Northwest Mexico. This crosssectional study included 307 Type 2 Diabetes Mellitus patients, who visited a Public hospital. Participants answered the PDRQ-9 as well as an adherence self-report scale individually using an electronic device inside a waiting room of a public health center. To investigate internal consistency and validity, reliability analysis and confirmatory factor analysis were performed. Our results demonstrated that the scale had acceptable reliability $(\alpha=0.95)$. A confirmatory factor analysis, using Maximum Likelihood method provided evidence of a good fit of the hypothesized one-factor model. All items presented an acceptable acceptable measurement weighs $(\lambda>0.5)$. These results show that the adaptation of the PDRQ-9 reproduced the factor structure found in the original study and demonstrated high internal consistency in a Northwest Mexican sample. This study suggests that this instrument is a valid and reliable instrument useful for measuring doctor-patient relationship in patients diagnosed with Type 2 Diabetes Mellitus.
\end{abstract}

Key words: Doctor-patient relationship; Psychometrics, validation, Diabetes, adaptation

DOI https://doi.org/10.36793/psicumex.v11i1.339

Recibido 24 de enero de 2020

Aceptado 05 de diciembre de 2020

Publicado 2 de junio de 2021 


\section{Introducción}

La relación médico-paciente puede ser definida como los comportamientos y actitudes que se desarrollan durante las interacciones clínicas (Blázquez-Manzano et al., 2012; Girón et al., 2002; KlausMichael, 2016). Este constructo se ha evaluado con la finalidad de prevenir problemas y mejorar la salud de pacientes (Linetzky et al., 2017). En este sentido, una buena relación se establece cuando el/la médico se comporta de forma amistosa, atenta y se muestra de forma empática al momento de comunicar sus resultados e indicaciones (Trujano et al., 2011). Así mismo, una buena relación incluye el grado de interés que presenta el/la médico al momento de responder las inquietudes de sus paciente y sus familiares, ofreciéndoles ayuda instrumental, emocional e informacional (Márquez, 2015).

Muchos estudios se han enfocado en aspectos negativos de la relación médico-paciente. Por ejemplo, previas investigaciones se han enfocado en la evaluación de la comunicación entre el/la médico y su paciente durante la consulta. Se ha encontrado que pacientes reportan una mala comunicación con su médico, a causa de una actitud autoritaria o distante (Celedón, 2016; Chacón et al., 2015). Este tipo de comunicación ha demostrado tener no solo consecuencias negativas en la relación médico-paciente sino también en la salud de pacientes (Trujano et al., 2011). Se ha demostrado que pacientes no solo son sensibles a la información brindada por su médico, sino que también al modo en el cual es desarrollada la consulta (DiMatteo et al., 1993; Finset et al., 2000; Kjaer y Finset, 2000; Mendoza et al., 2016). De esta manera una comunicación clara y amable ayuda a mejorar la salud de sus pacientes ya que incrementa el entendimiento de las indicaciones.

Una dificultad en la investigación de la relación entre médico-paciente es la perspectiva desde donde se aborda. Esta relación con frecuencia ha sido abordada bajo la perspectiva del/la profesional de la salud. En estas investigaciones se identifica al/la paciente como foco del problema cuando no existe una buena relación (Stokes, 2003). Recientes estudios han reconocido la importancia de la perspectiva del/la paciente 
(De Waard et al., 2018; Neuwirth, 1999; Swaminath, 2007). Se ha encontrado que la percepción que posea el/la paciente respecto a la relación que tiene con su médico y la calidad de esta está altamente relacionada con el poder curativo, es decir, se tendrá mayor adherencia al tratamiento prescrito (Nam y Song, 2014).

Basado en la perspectiva del/la paciente, lo que se logra es salir del modelo médico. El modelo médico considera al/la paciente como un actor pasivo, el cual no tiene ninguna autoridad ni acción en los encuentros terapéuticos y solo está en consulta para recibir un tratamiento sin alguna interacción. Por lo tanto, al considerar la percepción del/la paciente, se parte de la idea de que es un actor que participará de manera activa para el desarrollo de su tratamiento (Espigares y Orden, 2010).

Para evaluar la relación médico-paciente desde la perspectiva del/la paciente, Van der Feltz-Cornelis et al. (2004) desarrollaron un instrumento basado en el cuestionario Helping Alliance de Luborsky (Luborsky et al., 2012), una escala que utiliza la alianza terapéutica en psicoterapia. Inicialmente el instrumento constaba de 15 ítems que se agrupaban en 2 factores: uno relacionado con el/la médico y el otro con los síntomas del/la paciente. Debido a que el segundo factor sólo explicó 6 \% de la varianza, se eliminaron 6 ítems, quedando solo 9. Estos 9 ítems evalúan la relación médico-paciente, haciendo referencia a la comunicación, satisfacción y accesibilidad que pacientes perciben de su médico.

Previas investigaciones han mostrado que la relación médico-paciente se encuentra relacionada con la rapidez en la recuperación y la adherencia a los tratamientos médicos prescritos (Nam y Song, 2014). Por lo tanto, la importancia de dicha relación radica en la transferencia de conocimientos tanto teóricos como prácticos, de los cuales en gran medida depende el éxito terapéutico (Tamayo et al., 2016). En este sentido, la relación médico-paciente se considera como un factor de gran peso en el cumplimiento de las recomendaciones terapéuticas (DiMatteo et al., 1993; Froján y Rubio, 2005; Ruiz-Lurdury et al., 2017), además se asocia con la mejora de la salud física y mental, así como de la satisfacción del paciente y sus familiares (Vilató et al., 2015). 
El cuestionario relación médico-paciente o PDRQ-9, por sus siglas en inglés, ha sido validado y traducido para población española (Mingote et al., 2009) bajo el nombre de cuestionario de relaciones médico-paciente (CREM-P) en pacientes de medicina interna. Esta versión española del cuestionario fue validada a través de análisis de regresión logística y análisis factorial exploratorio mediante análisis de componentes principales y rotación varimax, en los que se encontraron buenos valores de confiabilidad y validez. De igual forma, se realizó una validación para población brasileña (Wollmann et al., 2018), con 133 usuarios/as de consulta médica, replicando el análisis factorial del original, se encontraron óptimos valores psicométricos en su consistencia interna. Sin embargo, no se ha validado la escala en una población latinoamericana de habla española.

Es de vital importancia contar con instrumentos válidos y confiables que permitan evaluar dicha relación para probabilizar la mejora en los tratamientos de los pacientes. Por ende, la mayoría de los estudios asociados con la relación médico-paciente se centran en pacientes con enfermedades crónicas, como es la diabetes, debido a que estos padecimientos se caracterizan por largos periodos de duración y prevalencia (García y Sánchez-Sosa, 2013). A pesar de esta gran importancia no se ha validado este instrumento en México y específicamente en pacientes de diabetes mellitus tipo 2. A partir de lo anterior, el objetivo de la investigación fue adaptar y validar el cuestionario de relación médico-paciente (Van der Feltz-Cornelis et al., 2004) en pacientes con diabetes mellitus tipo 2 en el noroeste de México. 


\section{Metodología}

\section{Participantes}

Participaron 307 pacientes con diabetes mellitus tipo 2, donde el $73 \%$ eran mujeres y el $27 \%$ fueron hombres, con una media de edad de 57 años $(D E=11.19$, rango $=46-69$ años $)$. En la presente muestra sobresalió el estado civil casado con el $88 \%$, seguido del viudo con $8 \%$, divorciado $3 \%$ y finalmente soltero con el $1 \%$ (ver Tabla 1 para estadísticos demográficos). Como criterio de inclusión, se consideraron a pacientes que presentaran al menos 1 año de diagnóstico y que no padecieran alguna complicación de la enfermedad.

\section{Tabla 1}

Análisis descriptivos

Frecuencia Porcentaje

\section{Sexo}

Hombre

85

$28 \%$

Mujer

221

$72 \%$

\section{Estado Civil}

Casado

271

$88 \%$

Divorciado

12

$3 \%$

Viudo

21

$8 \%$

Soltero

3

$1 \%$

\section{Media}

\section{Desviación}

Edad

57

11.19 


\section{Instrumentos}

\section{Relación médico-paciente}

Para medir la relación médico-paciente se aplicó una traducción del cuestionario Patient-Doctor Relationship Questionnaire de 9 preguntas (Van der Feltz-Cornelis et al., 2004). El cuestionario presenta una escala de tipo Likert de 5 puntos, donde 1 es totalmente en desacuerdo y 5 totalmente de acuerdo siempre. El instrumento tiene el objetivo de medir la percepción del paciente en cuanto a la comunicación, satisfacción y accesibilidad en el trato con el médico y el tratamiento. Dicho instrumento presentó un alfa de Cronbach aceptable $(\alpha=.95)$.

\section{Adherencia al tratamiento}

La adherencia al tratamiento también se midió a través de la aplicación de un cuestionario de elaboración propia (Fernández, 2020), el cual consta de 31 preguntas, con opción de respuesta tipo Likert de 4 puntos (opciones de respuesta: nunca, casi nunca, casi siempre y siempre). Teóricamente, el instrumento se encuentra constituido por 4 factores: medicación, ejercicio, dieta y consideraciones propias del tratamiento. 
El factor medicación consta de 7 reactivos (por ejemplo, "reactivo 5. Olvido tomar mis medicamentos"). El factor ejercicio presenta 8 reactivos en los cuales podemos encontrar como pregunta "Realizo la actividad física recomendada por mi médico" (reactivo 21). El factor dieta se encuentra conformado por 8 reactivos, los cuales hacen referencia a qué tanto el paciente se apega a los planes alimenticios adecuados para su salud (por ejemplo, "reactivo 10. Consumo alimentos no permitidos para mi padecimiento"). Finalmente, el factor consideraciones propias del tratamiento se compone de 8 reactivos. Estos hacen referencia a las implicaciones propias de la enfermedad y las necesidades de atención (por ejemplo, "reactivo 26. Realizo chequeos constantes de los síntomas de mi padecimiento"). El cuestionario presentó un alfa aceptable $(\alpha=.89)$.

\section{Traducción}

La adaptación del cuestionario se llevó a cabo a partir de su traducción al español y su retraducción al idioma original. La retraducción se comparó con el instrumento original. Se llevó a cabo un estudio piloto para verificar la pertinencia de cada ítem y su entendimiento en una muestra similar a la de interés del estudio, es decir, se aplicó en pacientes con diabetes mellitus tipo 2, en otro centro de salud público.

\section{Procedimiento}

\section{Aplicación de cuestionarios}

Se seleccionaron a los/as pacientes que asistían a consulta por diabetes, en un hospital público de la ciudad de Hermosillo, Sonora, México. Con ayuda del personal médico, se identificaron aquellos/as pacientes que cumplían con el criterio de inclusión establecido para la investigación. Los/as pacientes fueron invitados/as a participar de manera voluntaria. Con previo consentimiento informado, se les aplicó el cuestionario de manera digital e individual, con una duración aproximada de 15 minutos.

Ética 
La presente investigación tomó en cuenta las consideraciones éticas establecidas en el Código de ética del psicólogo (Sociedad Mexicana de Psicología, 2010). Adicionalmente se consideraron los lineamientos de Helsinki, los cuales están establecidos para la regulación de las investigaciones en seres humanos. Esta investigación fue sometida al Comité de Ética en Investigación dentro de la institución pública de salud en la cual se realizó la aplicación de instrumentos.

La solicitud de participación se realizó a través de invitaciones verbales, lo cual se llevó a cabo de manera personal, teniendo la aprobación se continuaba con la lectura del consentimiento informado. Se les informó sobre el objetivo de la investigación, de la supervisión de la investigación, se les dio un aviso de confidencialidad de los datos y se les agradeció por participar. Adicionalmente, se explicó que la investigación no produciría ningún riesgo hacia su persona y que existía la posibilidad de dejar el cuestionario en cualquier momento, sin ninguna consecuencia. Finalmente, no se ofrecieron incentivos inapropiados para obtener participantes de investigación. 


\section{Análisis de datos}

Los datos obtenidos se capturaron en el programa estadístico SPSS v23, donde se desarrollaron estadísticas descriptivas y alfa de Cronbach para evaluar la confiabilidad del instrumento. Para la realización del análisis factorial confirmatorio se utilizó el programa estadístico EQS v6, donde se utilizó el coeficiente de Mardia para determinar la existencia de normalidad multivariada de los datos.

De igual forma, se tomaron en cuenta indicadores prácticos y estadísticos como medida de ajuste, utilizando el método LS. Como indicador estadístico se tomó en cuenta la ji al cuadrado $\left(\chi^{2}\right)$, la cual su significancia es dependiente del tamaño de la muestra, por lo tanto, se tomó en cuenta la ji al cuadrado relativa $(<.5)($ Schumacker y Lomax, 2004).

Los indicadores prácticos contemplados fueron el índice de ajuste comparativo (CFI, por sus siglas en inglés), índice de ajuste normalizado de Bentler-Bonnet (NFI, por sus siglas en inglés), y finalmente, el índice de Tucker-Lewis (TLI, por sus siglas en inglés). De acuerdo con la literatura todos estos deben presentar un valor superior a .90 (Bentler, 2007). Por último, se utilizó el error de aproximación cuadrática media (RMSEA, por sus siglas en inglés) como medida absoluta de ajuste, cuyo valor debe de resultar $\leq .09$ (Browne y Cudeck, 1992).

Finalmente, para evaluar validez concurrente se realizó una correlación con adherencia al tratamiento. Para medir la validez convergente se utilizó la varianza media extraída (VME), la cual es satisfactoria si el valor es superior a .50 (Hair, 1995), además se consideró el coeficiente de McDonald con el mismo objetivo. Por último, para medir la validez discriminante, se calculó la raíz cuadrada de la varianza media extraída, en ella los valores esperados son una raíz cuadrada más grande que la covarianza entre los demás reactivos (Henseler et al., 2015).

\section{Resultados}


Los resultados muestran que la traducción al español de la escala de relación médico-paciente (PDRQ-9) es confiable y válida. Los ítems obtuvieron índices de confiabilidad aceptable $(\alpha=0.95)$. En la Tabla 2 se muestra el análisis de fiabilidad de los ítems incluidos en la escala, se puede observar que sin la eliminación de ningún reactivo el alfa se mantiene consistente $(\alpha \leq 0.95)$.

\section{Tabla 2}

Estadísticas de fiabilidad relación médico-paciente

\begin{tabular}{|c|c|c|c|c|}
\hline & & Media & $\begin{array}{l}\text { Desviación } \\
\text { estándar }\end{array}$ & $\begin{array}{l}\text { Alfa si se } \\
\text { elimina }\end{array}$ \\
\hline 1. & Mi médico me ayuda & 4.39 & .949 & .941 \\
\hline 2. & $\begin{array}{l}\text { Mi médico tiene suficiente tiempo } \\
\text { para mí }\end{array}$ & 4.31 & .985 & .942 \\
\hline 3. & Creo en mi médico & 4.22 & 1.085 & .941 \\
\hline & Mi médico me entiende & 4.34 & .981 & .941 \\
\hline 5 . & Mi médico se dedica a ayudarme & 4.30 & .960 & .942 \\
\hline & $\begin{array}{l}\text { Mi médico y yo estamos de acuerdo } \\
\text { sobre la naturaleza de mis síntomas }\end{array}$ & 4.44 & .842 & .942 \\
\hline 7. & Puedo hablar con mi médico & 4.54 & .850 & .944 \\
\hline & $\begin{array}{l}\text { Me siento contento con el tratamiento } \\
\text { de mi médico }\end{array}$ & 4.45 & .782 & .944 \\
\hline & $\begin{array}{l}\text { Siento que mi médico se encuentra } \\
\text { disponible para mí }\end{array}$ & 4.24 & 1.029 & .950 \\
\hline
\end{tabular}

Para el análisis factorial confirmatorio se consideró un solo factor que incluye los nueve ítems que hacen referencia a la comunicación, satisfacción y accesibilidad que se tiene en las consultas médicas. De igual forma los valores encontrados en el coeficiente de normalidad multivariada de Mardia (0.97) señalaron una distribución normal de los datos. La Figura 1 muestra los 9 ítems y sus pesos de medida a un solo factor. En congruencia con literatura previa, los resultados del análisis factorial revelaron una solución de un solo 
factor con bondad de ajuste aceptable $\left(\chi^{2}=50.87, \mathrm{gl}=26, \leq 1, p=.002 ; \mathrm{BNFI}=0.94 ; \mathrm{BNNFI}=0.96 ; \mathrm{CFI}\right.$ $=0.97 ;$ RMSEA $=0.09$ ).

\section{Figura 1}

\section{Modelo relación médico-paciente}

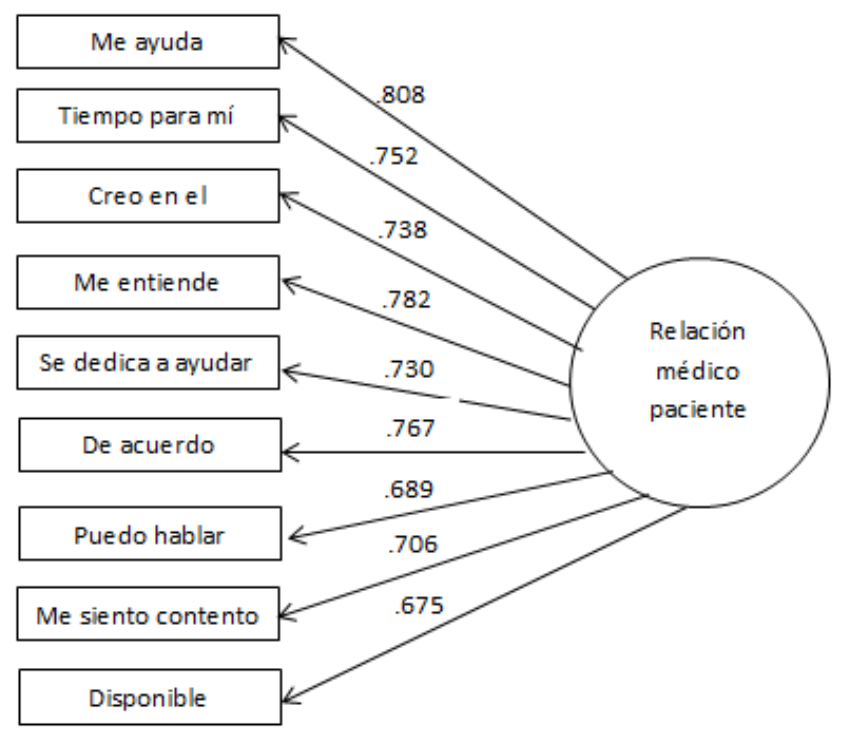

Nota. $\chi^{2}=50.87, \mathrm{gl}=26, p=.002, \mathrm{BNFI}=0.94 ; \mathrm{BNNFi}=0.96 ; \mathrm{CFI}=0.97 ; \mathrm{RMSEA}=0.09$.

Para medir validez de la escala se investigó la relación con un constructo teóricamente relevante, la adherencia al tratamiento. La correlación obtenida para el análisis de la validez concurrente entre médicopaciente y la adherencia al tratamiento fue significativa. Los resultados demostraron que existe una correlación positiva y estadísticamente significativa entre adherencia y la percepción de la relación que tiene el médico con su paciente ( $r=0.182, p=0.01)$, tal como se esperaba de acuerdo con la literatura.

Finalmente, se calculó la validez convergente de manera estandarizada a través de la varianza media extraída (VME) y la raíz cuadrada de la VME. De esta forma se demostró que el valor en el constructo es aceptable (.53). Así mismo, el coeficiente omega de McDonald mostro un valor aceptable (.94) y la raíz cuadrada del VME fue más alta que la correlación con otro constructo relacionado, adherencia al tratamiento 
(Tabla 3). Estos datos sugieren que esta versión en español demuestra tanto validez convergente como divergente.

\section{Tabla 3}

VME, coeficiente de McDonald y raíz cuadrada de la VME de la escala de relación médico-paciente

\begin{tabular}{lcccc}
\hline Indicadores & $\begin{array}{l}\text { Coeficientes } \\
\text { estandarizados al } \\
\text { cuadrado }\end{array}$ & $\begin{array}{l}\text { Coeficiente omega } \\
\text { McDonald }\end{array}$ & $\begin{array}{l}\text { Varianza media } \\
\text { extraída (VME) }\end{array}$ & $\begin{array}{l}\text { Raíz cuadrada de } \\
\text { la VME }\end{array}$ \\
\hline RMP-1 & .64 & & & \\
RMP-2 & .56 & & & \\
RMP-3 & .53 & & .72 \\
RMP-4 & .60 & .95 & & \\
RMP-5 & .53 & & & \\
RMP-6 & .57 & & & \\
RMP-7 & .46 & & & \\
RMP-8 & .49 & & & \\
RMP-9 & .44 & & & \\
\hline
\end{tabular}

\section{Discusión}

A manera de discusión, y de acuerdo con los resultados obtenidos en la presente investigación, podemos mencionar que el cuestionario Patient-Doctor Relationship Questionnaire (PDRQ-9) adaptado a población mexicana, específicamente en Sonora, México, es un instrumento confiable y válido para la evaluación de la relación médico-paciente. El instrumento presentó un alfa aceptable $(\alpha=.95)($ Nieva y Sorra, 2003), valores similares se han encontrado en la consistencia interna en el estudio original $(\alpha=.94)$ (Van der Feltz-Cornelis et al., 2004), al igual que en otras validaciones y traducciones $(\alpha=.95)$ (Wollmann et al., 2018). 
El instrumento evaluado también presenta validez concurrente, ya que se obtuvo una correlación positiva con la adherencia al tratamiento. Estos resultados son consistentes con otras investigaciones en donde la percepción de una buena relación con sus médicos, por parte de los pacientes, favorece el cumplimiento de las especificaciones señaladas por los especialistas de salud (Nam y Song, 2014; Tamayo et al., 2016); además, esta relación brinda beneficios significativos en la recuperación de los pacientes, mejorando su salud física, mental y satisfacción con su tratamiento (Vilató et al., 2015).

Así mismo, en congruencia con la literatura, el instrumento presentó bondad de ajuste aceptable destacando la obtención de 1 factor en el análisis factorial confirmatorio, en congruencia con la escala original (Van der Feltz-Cornelis et al., 2004). De la misma forma como se ha mantenido en las diversas validaciones y adaptaciones que se han encontrado en la literatura se mantuvieron los 9 reactivos originales, conformando el factor único de la variable (Mingote et al., 2009; Wollmann et al., 2018). En este sentido, nuestra investigación fortalece las propiedades psicométricas de la escala aportando un instrumento que considere la percepción del paciente acerca de su médico y la calidad de la relación con dicho trabajador de la salud. Finalmente, se obtuvo validez convergente y divergente, ya que obtuvimos una varianza media extraída (VME) mayor a .5 (.53) y la raíz cuadrada de la VME presentó un valor mayor que el obtenido en la correlación entre los constructos y un coeficiente omega de McDonald mayor a .70 (.94).

Como la literatura puntualiza, la relación médico-paciente es fundamental para la adherencia al tratamiento. Esto se debe a que durante las consultas médicas se pueden manifestar las interrogantes, expectativas, necesidades, opiniones acerca de los tratamientos subscritos, de tal forma que el paciente se convierte en un agente activo, lo cual beneficia al cumplimiento de los mismos (Chacón et al., 2015; Linetzky et al., 2017; Sandín et al., 2012; Vilató et al., 2015).

A pesar de la relevancia de los resultados obtenidos en la presente investigación, es necesario considerar como limitación el hecho de que haya sido una muestra por conveniencia y un diseño transversal. 
Esto pone en evidencia la posibilidad de no poder generalizar los resultados obtenidos. Por lo tanto, se sugiere continuar con esta línea de investigación con la finalidad de replicar los resultados obtenidos, con un mayor número de participantes y un muestreo de tipo aleatorio. De esta manera, se pudiera incrementar la validez externa y reafirmar la composición psicométrica del instrumento. Finalmente, se sugiere la comparación de resultados con muestras de diversos hospitales, considerando hospitales públicos, así como privados, para evaluar si existen diferencias estadísticamente significativas entre dichas muestras.

El presente estudio presenta resultados sobre la validez y confiablidad de una escala de la relación entre paciente y médico. Debido a la importancia de este constructo en la mejora de la adherencia al tratamiento de los pacientes, es importante la identificación de las variables relacionadas que permitan la generación de programas de intervención y promoción que centren la acción en la relación médico-paciente. De igual forma, es importante que los médicos tengan conciencia de su función y relevancia en el manejo de las interacciones durante las consultas médicas y su efecto en la adherencia al tratamiento y por la tanto en la salud de los pacientes (Linetzky et al., 2017). Finalmente, esta investigación aporta un instrumento válido y confiable para la medición de la variable relación médico-paciente, el cual ha sido adaptado al contexto de la población de interés, siendo de fácil aplicación considerando las particularidades de la muestra, con tan solo 9 ítems.

\section{Conflicto de intereses}

Los autores declaran no tener conflictos de intereses.

\section{Referencias}

Bentler, P. (2007). On tests and indices for evaluating structural models. Personality and Individual Differences, 42(5), 825-829. 
Blázquez-Manzano, A., Feu-Molina, S., Ruíz-Muñoz, E. y Gutiérrez-Caballero, J. (2012). Importancia de la comunicación interpersonal en relación médico-paciente en atención primaria. Rev Esp Comun Salud., 3(1), 62-76.

Browne, M. y Cudeck, R. (1992). Alternative ways of assessing model fit. Sociological Methos and Research, 21(2), 230-258.

Celedón L, C. (2016). Relación médico paciente. Revista de Otorrinolaringología y Cirugía de Cabeza y Cuello, 76(1), 51-54. https://doi.org/10.4067/S0718-48162016000100007

Chacón, J., Sandoval, D., Muñoz, R. y Romero, T. (2015). Evaluación del control de la presión arterial y la adherencia terapéutica en hipertensos seguidos en el Programa de Salud Cardiovascular (PSCV): asociación con características clínicas, socioeconómicas y psicosociales. Revista Chilena de Cardiología, 34(1), 18-27. https://doi.org/10.4067/S0718-85602015000100002

De Waard, C. S., Poot, A. J., den Elzen, W. P. J., Wind, A. W., Caljouw, M. A. A. y Gussekloo, J. (2018). Perceived doctor-patient relationship and satisfaction with general practitioner care in older persons in residential homes. Scandinavian Journal of Primary Health Care, 36(2), 189-197. https://doi.org/10.1080/02813432.2018.1459229

DiMatteo, M., Sherbourne, C. y Hays, R. (1993). Physicians' characteristics influence patient' adherence to medical treatment: Results from the medical outcome study. Health Psychology, 12, 93-102.

Espigares, L. y Orden, P. (2010). La relación médico paciente desde la perspectiva del paciente. VI Jornadas de Sociología de la UNLP. Universidad Nacional de La Plata. Facultad de Humanidades y Ciencias de la Educación. Departamento de Sociología, La Plata. https://www.aacademica.org/000-027/624

Finset, A., Smedstad, L. y Ogar, B. (2000). Physician-patient interaction and coping with cancer: The doctor as informer or supporter? Journal of Cancer educ, 12, 174-178. 
Froján, M. y Rubio, R. (2005). Discrimant analysis of treatment adherence in insulin-dependent diabetes mellitus. Psychology in Spain, 9(1), 41-48.

García, R. y Sánchez-Sosa, J. (2013). Efectos de la entrevista motivacional en el tratamiento de la diabetes mellitus tipo 2. Psicología y Salud, 23(2), 183-193.

Girón, M., Beviá, B., Medina, E. y Simón-Talero, M. (2002). Calidad de la relación médico paciente y resultados de encuentros clínicos en atención primaria de Alicante. Revista Española de Salud Pública, 76, 561-575.

Hair, J. (1995). Multivariate data analysis with reading. Prentice Hall.

Henseler, J., Ringle, C. M. y Sarstedt, M. (2015). A new criterion for assessing discriminant validity in variance-based structural equation modeling. Journal of the Academy of Marketing Science, 43(1), 115-135. https://doi.org/10.1007/s11747-014-0403-8

Kjaer, P. y Finset, A. (2000). Trait anxiety and reactions to patient-centered and Doctor-centered styles of commnication: An experimental study. Psychosomatic Medicine, 62, 33-39.

Klaus-Michael, T. (2016). Patient-Doctor Relationship in Dermatology: From Compliance to Concordance. Medical Journals Limited, 96(5), 25-29.

Linetzky, B., Jiang, D., Funnell, M. M., Curtis, B. H. y Polonsky, W. H. (2017). Exploring the role of the patient-physician relationship on insulin adherence and clinical outcomes in type 2 diabetes: Insights from the MOSAIc study. Journal of Diabetes, 9(6), 596-605. https://doi.org/10.1111/17530407.12443

Luborsky, L., Barber, J. P., Siqueland, L., Johnson, S., Najavits, L. M., Frank, A., \& Daley, D. (2012). Helping Alliance Questionnaire-II [Data set]. American Psychological Association. https://doi.org/10.1037/t07504-000 
Márquez, A. (2015). Empatía y satisfacción de la “Relación médico-paciente” en la UMF No. 66 de Xalapa, Veracruz. Universidad Veracruzana.

Mendoza, C., Ramos-Rodríguez, C. y Gutierrez, E. (2016). Relación médico-paciente percibida por usuarios de consultorios externos de un hospital de Lima, Perú. Horizonte Médico, 16(1), 14-19.

Mingote, A., Moreno, B., Rodríguez, R., Gálvez, M. y Ruiz, P. (2009). Validación psicométrica de la versión española del Cuestionario de Relaciones Médico-Paciente (CREM-P). Actas Esp Psiquiatr, 37(2), 94-100.

Nam, S. y Song, Y. (2014). Role of Self-Efficacy in the Relationship Between Patient-Provider Relationships and Psychological Insulin Resistance Among Patients with Type 2 Diabetes. J Contemp Diabetes Res, 1(1), 1-15.

Neuwirth, Z. E. (1999). An essential understanding of physician-patient communication. Part II. The Journal of Medical Practice Management: MPM, 15(2), 68-72.

Nieva, V. y Sorra, J. (2003). Safety culture assessment: A tool for improving patient safety in healthcare organizations. Quality Safe Health Care, 12, 17-23.

Ruiz-Lurdury, R., Torres-Torres, L. y Velandia-Novoa, K. (2017). Relación médico-paciente y adherencia al tratamiento. Ciencia y Humanismo en la salud, 4(1), 29-37.

Sandín, M., Río, I., y Larraz, R. (2012). Diseño de un catálogo de recursos online para la mejora de la comunicación sanitario-paciente inmigrante. Revista Española de Comunicación en Salud, 3(1), 3848.

Schumacker, R., y Lomax, R. (2004). A beginer's guide to structural equation modeling. Psychology Press. Sociedad Mexicana de Psicología (2010). Código de ética del psicólogo. Trillas.

Stokes, T. (2003). Breaking up is never easy: GPs' accounts of removing patients from their lists. Family Practice, 20(6), 628-634. https://doi.org/10.1093/fampra/cmg602 
Swaminath, G. (2007). Doctor-patient communication: Patient perception. Indian Journal of Psychiatry, 49(3), 150-153. https://doi.org/10.4103/0019-5545.37309

Tamayo, G., Máynez, L. y Rodas, J. (2016). Decisión terapéutica en la relación médico-paciente desde la perspectiva del paciente. Utilidad del análisis con modelos matemáticos de decisión. Acta Medica Grupo Ángeles, 14(4), 220-229.

Trujano, R., Vega, Z., Nava, C. y Saavedra, K. (2011). Interacción médico-paciente y su relación con el control del padecimiento en enfermos crónicos. Liberabit. Revista de Psicología, 17(2), 223-230.

Van der Feltz-Cornelis, C. M., Van Oppen, P., Van Marwijk, H. W. J., De Beurs, E. y Van Dyck, R. (2004). A patient-doctor relationship questionnaire (PDRQ-9) in primary care: Development and psychometric evaluation. General Hospital Psychiatry, 26(2), 115-120. https://doi.org/10.1016/j.genhosppsych.2003.08.010

Vilató, L., Martín, L. y Pérez, I. (2015). Adherencia terapéutica y apoyo social percibido en personas que viven con VIH/sida. Revista Cubana de Salud Pública, 41(4), 620-630.

Wollmann, L., Hauser, L., Mengue, S. S., Roman, R. y Van der Feltz-Cornelis, C. M. (2018). Cross-cultural adaptation of the PatientDoctor Relationship Questionnaire (PDRQ-9) in Brazil. Revista de Saúde Pública, 52, 71. https://doi.org/10.11606/S1518-8787.2018052000380

\footnotetext{
Cómo citar este artículo: Fernández Castillo, K. N., Corral Frías, N. S., \& Valenzuela Hernández, E. R. (2021). Validación y adaptación al español del cuestionario de relación médico-paciente en pacientes de diabetes mellitus tipo 2 en el norte de México (PDRQ-9). Psicumex, 11(1), 1-19, e339. https://doi.org/10.36793/psicumex.v1111.339
} 